\title{
Construction and Analysis of Two Baryon Correlation functions
}

\section{Kostas Orginos ${ }^{* \dagger}$}

Department of Physics, College of William and Mary, Williamsburg, VA, 23187, USA

Jefferson Laboratory, Newport News, VA, 23606, USA

E-mail: kostas@wm.edu

The signal to noise ratio of baryon two point functions grows exponentially with Euclidean time hindering reliable extraction of the energy levels of the system. The problem becomes more acute in the case of two or more baryon correlation functions. For this reason special care is needed in both constructing, and analyzing two point functions of multi-baryon systems. In this talk I discuss methods for extracting the energy levels associated from two point functions for two baryon systems.

The XXVIII International Symposium on Lattice Filed Theory

June 14-19,2010

Villasimius, Sardinia Italy

\footnotetext{
* Speaker.

${ }^{\dagger}$ This work was supported by DOE DE-FG02-04ER41302, NSF CCF-0728915 and the DOE DE-AC0506OR23177 under which JSA operates the TJNF.
} 


\section{Motivation}

Lattice calculations of properties of two baryon systems are now beginning to receive serious attention. The current goal of such calculations is obtaining from first principles the elastic scattering phase shifts of two baryon systems as well as the spectrum of their bound states. The basic approach for such calculations is very well known [1]. However, only recently computational resources are beginning to become sufficient for tackling the problem.

The main computational task is to obtain the spectrum of a two baryon system in a finite box. Given than the characteristic scales in nuclear physics are low relative to the $1 \mathrm{GeV}$ scale of QCD interactions, we need to obtain the finite volume energy eigenstates of the two particle system with rather high precision. In most cases the effects of baryon-baryon interactions are of the order of few $\mathrm{MeV}$. For this reason one needs very precise lattice calculations with very good control of all systematics in order to have reliable determinations of baryon-baryon scattering phase shifts from Lattice QCD. Furthermore, the spacing between neighboring energy levels of the two particles in the box is rather dense further complicating the extraction of the ground state energy. In order to understand the scale of severity of this problem one can assume for that the interaction energy between two identical baryons is negligible. In this case the two particle spectrum in the box would be

$$
E(n)=2 \sqrt{m^{2}+\left(\frac{2 \pi}{L}\right)^{2} \vec{n} \cdot \vec{n}}
$$

where $m$ is the mass of the single baryon, $\vec{n}$ is the wave number vector $\vec{n}=\left(n_{x}, n_{y}, n_{z}\right)$ for the relative momentum of the two particles and $L$ is the size of the box. In order to isolate the ground state energy using single exponential fits one would have to calculate the correlation function at time separations $\Delta t$ much larger that the the energy level separation $\Delta E$. If we assume that the relative contamination from excited states should be smaller or equal to $10^{-2}$, and that all states couple to our interpolating fields with roughly the same weights, we can estimate the required $\Delta t$ using typical values for $m \sim 1 \mathrm{GeV}$ and $L \sim 3 \mathrm{fm}$. In Figure 1 we plot the estimated time separation $\Delta t$ for suppressing the contamination from each of the lowest six excited states to the ground state by a factors of $10^{-2}$. From these considerations separations of about $6 \mathrm{fm}$ seem to be required to achieve an isolation of the ground state for a two baryon system. Recent lattice calculations of rather high statistics [2] demonstrated that it is hard to resolve the signal for two nucleons for distances more than $2 \mathrm{fm}$. For baryonic systems for sufficiently large time separations the noise to signal ratio grows exponentially due to the fact that pions are much lighter than baryons [3]. For these reasons, it is clear that special care has to be given for reliable extraction of the ground state and low lying states of two baryon systems.

Fortunately, the situation is a little better than the toy example seems to imply. This is because in some cases one can suppress the coupling to excited states using carefully chosen interpolating fields. Generally, smeared sources seem to accomplish this suppression for single particle correlation functions. However, as it was shown in [4] appropriately used variational methods can have a larger effective energy gap $\Delta E$ that determines the relevant time separation $\Delta t$ for suppressing contaminations from excited states. In this approach one uses a basis of interpolating fields and constructs a symmetric matrix of correlation functions. Then a generalized eigenvalue problem determines the energies and the "optimal" interpolating fields for the lowest few energy levels that 


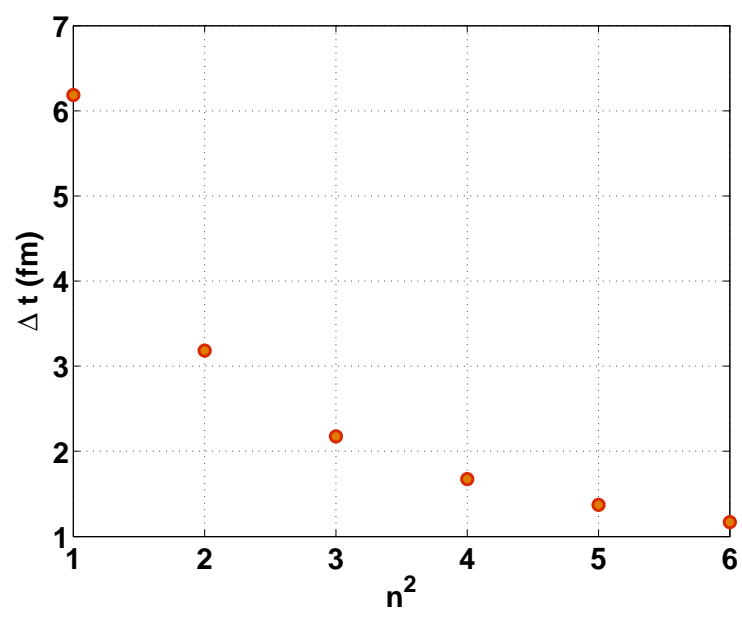

Figure 1: Required time separation for suppressing the contamination from excited states to the ground state by $10^{-2}$.

couple to the original basis of interpolating fields. From Figure 1, we can estimate that if the lowest six states of a two baryon system are captured in the basis of interpolating fields we use, then we have a hope of isolating the ground state at time separations of $1 \mathrm{fm}$ or so. At this time separation it has been shown [2] that the relevant correlation functions can be computed reliably.

Unfortunately, constructing a large symmetric matrix of correlation functions for two baryon systems is computationally expensive. The plane wave two particles interpolating fields are known to couple well to the scattering states of weakly interacting two particle systems [1]. However, these interpolating fields require some variant of "all-to-all" quark propagators in order to be constructed. For this reason we would like to explore the possibility of a variational-like approach that works with a non-symmetric matrix of correlators, as those used in $[2,5]$.

\section{The non-symmetric generalized eigenvalue problem}

Lets now assume that we have a non-symmetric matrix $C_{i j}$ of correlation functions is constructed from two sets of interpolating operators: $\mathscr{O}_{i}$ are the annihilation operators and $\tilde{\mathscr{O}}_{i}$ are the creation operators.

$$
C_{i j}(t)=\left\langle\mathscr{O}_{i}(t) \tilde{\mathscr{O}}_{j}^{\dagger}(0)\right\rangle=\left\langle 0\left|\mathscr{O}_{i} e^{-\hat{H} t} \tilde{\mathscr{O}}_{j}^{\dagger}\right| 0\right\rangle,
$$

where $\hat{H}$ is the Hamiltonian. This matrix can be decomposed as a sum over all energy eigenstates of the Hamiltonian $\hat{H}$

$$
C_{i j}(t)=\sum_{n=1}^{\infty} Z_{i n} \tilde{Z}_{j n}^{*} e^{-E_{n} t}
$$

The eigenstates of the Hamiltonian $|n\rangle$ are

$$
\hat{H}|n\rangle=E_{n}|n\rangle, Z_{i n}=\left\langle 0\left|\mathscr{O}_{i}\right| n\right\rangle . \tilde{Z}_{i n}^{*}=\left\langle n\left|\tilde{\mathscr{O}}_{i}^{\dagger}\right| 0\right\rangle
$$

In matrix form the spectral representation of the correlation function matrix can be written as

$$
C(t)=Z \Lambda \tilde{Z}^{\dagger},
$$


with

$$
\begin{gathered}
\Lambda=\operatorname{diag}\left(e^{-E_{1} t}, e^{-E_{2} t}, \cdots\right) \equiv \operatorname{diag}\left(\lambda_{1}(t), \lambda_{2}(t), \cdots\right) . \\
\lambda_{i}(t) \equiv e^{-E_{i} t}
\end{gathered}
$$

In the remaining we will assume that the energy levels are ordered

$$
E_{1}<E_{2}<E_{3} \cdots
$$

Degenerate energy levels introduce complexities that we will avoid for now.

If we now assume that the system has $N$ energy levels and we use a set of $N$ linearly independent interpolating fields $\mathscr{O}_{i}$ and $N$ linearly independent interpolating fields $\tilde{\mathscr{O}}_{i}$ we can compute all the energy levels from the correlator matrix $C_{i j}$. One way of doing this is by constructing two matrices $Q$ and $\tilde{Q}$ so that

$$
Q^{\dagger}=(Z)^{-1} \quad, \quad \tilde{Q}=\left(\tilde{Z}^{\dagger}\right)^{-1}
$$

This is always possible since $Z$ and $\tilde{Z}$ are $N \times N$ matrices with a non-vanishing determinants. The fact that $\mathscr{O}_{i}$ and $\tilde{\mathscr{O}}_{i}$ are linearly independent guarantees that $\operatorname{det}(Z) \neq 0$ and $\operatorname{det}(\tilde{Z}) \neq 0$. Using this property we can now extract the energies by

$$
Q^{\dagger} C(t) \tilde{Q}=\Lambda(t)
$$

It turns out that $\tilde{Q}$ is a solution to a generalized eigenvalue problem:

$$
C(t) \tilde{Q}=Q^{\dagger} \Lambda(t)=Z \Lambda\left(t_{0}\right) \Lambda\left(t-t_{0}\right)=Z \Lambda\left(t_{0}\right) \tilde{Z}^{\dagger} \tilde{Q} \Lambda\left(t-t_{0}\right)=C\left(t_{0}\right) \tilde{Q} \Lambda\left(t-t_{0}\right)
$$

Note that the factorization of $\Lambda(t)=\Lambda\left(t_{0}\right) \Lambda\left(t-t_{0}\right)$ will not hold when periodic images need to be included due to boundary conditions in the finite time direction. Equivalently the columns $\tilde{q}_{i}$ of the matrix $\tilde{Q}$ are the generalized right eigenvectors that satisfy

$$
C(t) \tilde{q}_{i}=C\left(t_{0}\right) \tilde{q}_{i} \lambda_{i}\left(t-t_{0}\right)
$$

Similarly the matrix $Q$ can be determined as the solution of the left generalized eigenvalue problem

$$
Q^{\dagger} C(t)=\Lambda\left(t-t_{0}\right) Q^{\dagger} C\left(t_{0}\right)
$$

Equivalently the columns $q_{i}$ of the matrix $Q$ are the generalized right eigenvectors that satisfy

$$
q_{i}^{\dagger} C(t)=\lambda_{i}\left(t-t_{0}\right) q_{i}^{\dagger} C\left(t_{0}\right)
$$

Given the above discussion the algorithm for determining the energy levels contributing to the correlation function is simple. We first solve the generalized eigenvalue problem of Eq. 2.10 and then determine the energy levels by taking the logarithm of the eigenvalues $\lambda_{i}$ :

$$
E_{i}=\frac{1}{\tau} \log \left(\frac{\lambda_{i}\left(t-t_{0}\right)}{\lambda_{i}\left(t+\tau-t_{0}\right)}\right)
$$

In this simple example the answer is independent of $t_{0}$ and $t$ and is exactly the eigenvalues of the Hamiltonian that contribute to the correlator. It can be shown that deviations from this ideal case, where the basis of interpolating fields has dimension equal to the dimension of the space the Hamiltonian acts on, behaves similarly to the case of symmetric correlation matrices [6]. 


\section{Construction of non-symmetric basis of interpolating fields}

The NPLQCD collaboration has been using correlation functions for two baryon systems that are constructed from a single smeared quark source resulting a single $\tilde{\mathscr{O}}^{\dagger}$ creation interpolating field for each channel. Having computed the smeared quark propagators, one can then build several annihilation interpolating fields, including the plane wave two particle states that would be the eigenstates of the Hamiltonian in the absence of interactions. This matrix of correlators is nonsymmetric due to the difference in creation and annihilation basis both in kind and in size. Yet, we can still proceed with a variational-like procedure like the one described in Section 2. From our initial basis of interpolating fields we can generate a new larger set by using time translation. In Euclidean space we translate forward in time the annihilation interpolating fields and backward in time the creation interpolating fields as following:

$$
\begin{aligned}
& \mathscr{O}_{i}(t+m \tau)=e^{m \tau H} \mathscr{O}_{i} e^{-m \tau H} \\
& \tilde{\mathscr{O}}_{i}(t-m \tau)=e^{-m \tau H} \mathscr{O}_{i} e^{m \tau H}
\end{aligned}
$$

where $\tau$ is a choice for a time step and $m$ is an integer. Using time translation one can construct several new interpolating fields by increasing the integer $m$. With this in mind we can always take a correlator matrix with un-equal dimensions and convert it to a square matrix by sufficient number of time translations of the existing interpolating fields. For doing so, we only need to time translate the already computed matrix of correlators. This is because

$$
\left\langle 0\left|\mathscr{O}_{i}(t+m \tau) \tilde{\mathscr{O}}_{i}(t-n \tau)\right| 0\right\rangle=C_{i j}(t+n \tau+m \tau) .
$$

Using time translation we can now work with a new interpolating correlator square matrix $K_{i j}{ }^{1}$.

With this enlarged time translated basis of interpolating fields we now have a square matrix of correlators from which energy levels can be extracted as described in Section 2 by solving the generalized eigenvalue problem

$$
K(t) \tilde{q}_{i}=K\left(t_{0}\right) \tilde{q}_{i} \lambda_{i}\left(t-t_{0}\right), q_{i}^{\dagger} K(t)=\lambda_{i}\left(t-t_{0}\right) q_{i}^{\dagger} K\left(t_{0}\right)
$$

One complication that arises is the fact that the interpolating fields we end up with are not linearly independent. For example, if in the time range we work in, the size of the resulting basis is larger than the number of energy levels significantly contributing to the correlators, then the interpolating fields are not linearly independent and one of the basic assumptions in Section 2 will be violated. This problem is avoided by using singular value decomposition (SVD) to solve the generalized eigenvalue problem. If we consider the SVD of $K\left(t_{0}\right)$ then

$$
K\left(t_{0}\right)=U \Sigma V^{\dagger}
$$

with $S=\operatorname{diag}\left(\sigma_{i}\right)$ a diagonal matrix with the singular values of $K\left(t_{0}\right)$ in the diagonal and $U$ and $V$ unitary matrices. We now define $S^{\prime}$ as the sub-matrix of $S$ containing only the non-vanishing singular values (in practice we include the singular values $\sigma_{i}$ that are larger than a pre-decided

\footnotetext{
${ }^{1}$ As it will be obvious from the subsequent analysis the matrix $K$ could remain non-square. The resulting generalized eigenvalue problem can be solved with using SVD.
} 

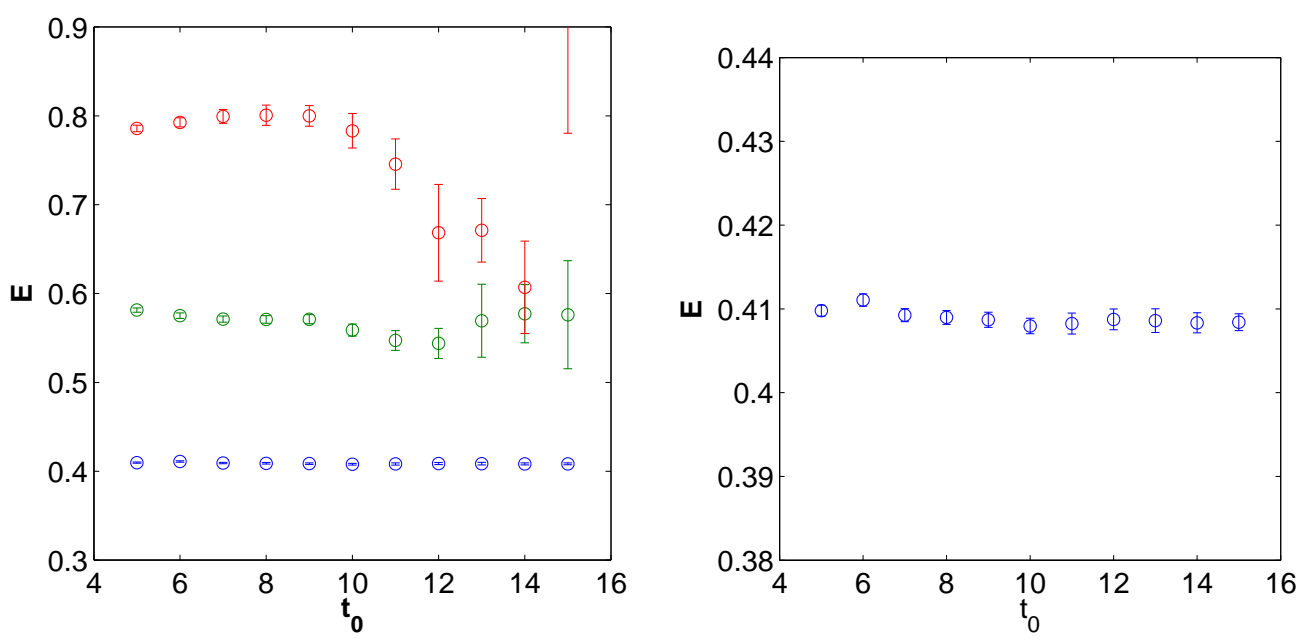

Figure 2: Energy levels of the proton-proton system int the ${ }^{1} S_{0}$ channel as a function of $t_{0}$. On the left the three energy levels extracted from fitting the eigenvalues to single exponentials for each $t_{0}$ in the range of $\left(t_{0}+1,2 t_{0}\right)$. On the right a magnification of the ground state is plotted.

tolerance). We also define $U^{\prime}$ and $V^{\prime}$ to be the columns of $U$ and $V$ that correspond to the selected non-vanishing singular values. We can now project the creation and annihilation operators to new sets that are linearly independent using

$$
\tilde{\Omega}_{i}=\sum_{k}\left[\Sigma^{\prime-1 / 2} V^{\prime \dagger}\right]_{i k} \tilde{\mathscr{O}}_{k}, \Omega_{i}=\sum_{k}\left[\Sigma^{\prime-1 / 2} U^{\prime \dagger}\right]_{i k} \mathscr{O}_{k}
$$

With the above projection the new correlation matrix $K^{\prime}(t)$ becomes

$$
K^{\prime}(t)=\Sigma^{\prime-1 / 2} U^{\prime \dagger} K(t) V^{\prime} \Sigma^{\prime-1 / 2}
$$

The dimension of $K^{\prime}(t)$ is now equal to the number of non-vanishing singular values. In addition $K^{\prime}\left(t_{0}\right)=\mathbf{1}$. We can now re-write the generalized eigenvalue problem as regular eigenvalue problem using

$$
K^{\prime}(t) \tilde{\omega}_{i}=\tilde{\omega}_{i} \lambda_{i}\left(t-t_{0}\right), \omega_{i}^{\dagger} K^{\prime}(t)=\lambda_{i}\left(t-t_{0}\right) \omega_{i}^{\dagger}
$$

This is now a regular non-symmetric matrix eigenvalue problem that can be solved for the left $(\omega)$ and right $(\tilde{\omega})$ eigenvectors and the eigenvalues $\lambda_{i}\left(t-t_{0}\right)$. The eigenvalues can now be used to extract the desired energy levels using eq. 2.14 , or fits to single exponentials.

The idea of using multiple time shifts of a signal to recast the problem of estimating the contributing frequencies into a generalized eigenvalue problem has been used before in signal processing (for example [7, 8]). In [7, 8] SVD was used to obtain the number as well as the values of the contributing frequencies. In the above I have generalized the method to the case of a matrix of correlators as well as established the connection to the creation and annihilation operators of the underlying quantum field theory. This connection allows for use of the obtained eigenvectors and projection operators with the analysis of three point functions for extracting hadronic matrix elements [9]. 


\section{An example}

In order to demonstrate how the method works we use correlators for a two proton system in the ${ }^{1} S_{0}$ channel provided by NPLQCD collaboration. The original correlators had a sigle smeared creation interpolating field and three annihilation interpolating fields. The annihilation interpolating fields had protons at zero relative momentum with quarks smeared in both protons, not smeared in both protons and smeared in one proton only. The gauge fields where $2+1$ clover anisotropic fermions with anisotropy 3.5 on a $24^{3} \times 128$ lattice. The pion mass was roughly $390 \mathrm{MeV}$. For details on these ensembles see [2,5]. Form the original 3 by 1 matrix of correlators we constructed a 3 by 3 matrix of correlators using the time shifting described in Sec. 3 . The resulting 3 eigenvalues were fitted to single exponentials in the interval $\left(t_{0}+1,2 t_{0}\right)$. If Fig. 2 we plot the resulting masses for the three energy levels as a function of $t_{0}$. The extracted energy levels should be independent of $t_{0}$. For the ground state we can see that such independence is achieved within statistical errors for $t_{0}>10$ (errors where computed under jackknife). For the excited states we see significant variation with $t_{0}$ indicating contaminations from other missing states. Furthermore, given the small size of the initial basis of interpolating fields several low lying states are not observed.

\section{Conclusions}

In conclusion, the described method seems as an interesting alternative to the symmetric generalized eigenvalue problem used for extracting energy levels from correlator matrices. Further analysis of the sensitivity of the extracted energy levels to both statistical fluctuations as well as contaminations from excited states is needed in order to establish the reliability of the method. Such analysis is underway.

\section{References}

[1] M. Luscher, U. Wolff, Nucl.Phys. B339, 222 (1990)

[2] S.R. Beane et al. (NPLQCD Collaboration), Phys.Rev. D81, 054505 (2010), 0912.4243

[3] G.P. Lepage, Boulder TASI'89 p. 97 (1989)

[4] B. Blossier, M. Della Morte, G. von Hippel, T. Mendes, R. Sommer, JHEP 0904, 094 (2009), 0902.1265

[5] S.R. Beane et al., Phys.Rev. D80, 074501 (2009), 0905.0466

[6] K. Orginos et al., in preparation (2011)

[7] Y. Hua, T.K. Sarkar, Antennas and Propagation 37, 229 (1989)

[8] T. Sarkar, O. Pereira, IEEE Antennas and Propagation Magazine 37, 48 (1995)

[9] C. Aubin, K. Orginos (2010), arXiv:1010.0202 